\title{
Detection of Photopolymerization Processes of Diacetylenic Langmuir-Blodgett Films on a Core of Plastic Optical Fiber as a Light-Emitting Diode Signal Change
}

\author{
Nobuyuki Higashi ${ }^{\dagger}$, Tomoki Kato, and Masazo NiWA ${ }^{\dagger}$ \\ Department of Molecular Science \& Technology, Faculty of Engineering, Doshisha University, \\ Kyotanabe, Kyoto 610-0321, Japan
}

(Received April 3, 1998)

\begin{abstract}
KEY WORDS Diacetylenic Lipid / Polydiacetylene Assembly / Surface Monolayer / Langmuir-Blodgett Film / Plastic Optical Fiber / Light-Emitting Diode /
\end{abstract}

We have demonstrated previously the preparation of plastic optical fibers (POF) modified with polyioncomplexed multibilayer assemblies, as the cladding, and their laser-light responsiveness. ${ }^{1-3}$ The modified POFs, through which the laser-light passes continuously, have exhibited drastic changes in the laser optical intensity caused by a crystal-to-liquid crystal phase transition of the bilayer component and by some chemical perturbations, resulting from the variation in refractive index of the membrane phase.

In this paper, we describe the photopolymerization of diacetylenic Langmuir-Blodgett (LB) films deposited on the core of POF and detectability of such polymerization processes based on an optical signal measurement of light-emitting diode (LED) with a wavelength of $660 \mathrm{~nm}$ (Figure 1). The topotactic polymerization of diacetylene is well-known to be acutely sensitive to the molecular order of assemblies. O'Brien et al. have demonstrated that the photopolymerization of cast films from bilayer dispersions of a diacetylenic lipid (1) successfully proceeds below the phase transition temperature. ${ }^{4-6}$ More recently, some reports ${ }^{7,8}$ have been appeared on polymerization properties and morphology of a new type of ultrathin polydiacetylene films prepared by self-assembly techniques. The resultant polydiacetylene films have the unique optical properties; the conjugated backbone of alternating double and triple bonds causes intense absorptions in the visible spectrum. Here it occurs to us that the light of LED through the POFs would respond to the polymerization, since the highly conjugated polydiacetylene is known to show an absorption maximum at around 600-640 $\mathrm{nm}^{9}$ which is close to the emission maximum of the LED $(660 \mathrm{~nm})$.

\section{EXPERIMENTAL}

The diacetylene ammonium lipid (1) was prepared according to the same manner to that of $\mathrm{O}^{\prime}$ Brien et al. ${ }^{4}$

The monolayers were obtained by spreading a benzene solution of 1 (about $1 \mathrm{mg} \mathrm{ml}^{-1}$ ) on purified water (Milli$\mathrm{Q}$ system, Millipore Ltd.). Twenty minutes after spreading, the monolayer was compressed with a rate of 1.20 $\mathrm{cm}^{2} \mathrm{~s}^{-1}$. On the case of the compression-expansion cycle, the monolayer was compressed up to a certain

\footnotetext{
† To whom all correspondence should be addressed.
}

surface pressure and then expanded continuously to the original barrier position with the same rate. Whilhelmy's plate method and a Teflon-coated trough with a micro-processor-controlled from balance, FSD50 (USI System Ltd.), with a precision of $0.01 \mathrm{mN} \mathrm{m}^{-1}$ were used for surface pressure measurements.

The fluorescence microscopy equipment (USI System Ltd.) consisted of a fluorescence microscope with a video-recording-display unit and a thermostated trough $\left(250 \mathrm{~cm}^{2}\right)$ which was placed below the objective of the microscope. Octadecanoyl Rhodamine B of $0.5 \mathrm{~mol} \%$ as a fluorescent probe was added to the $\mathbf{1}$ solution before spreading.

The POF (TB 500, Asahi Chemical Industry Co., Ltd.) used in this study consists of the core (refractive index, 1.49) with a diameter of $500 \mu \mathrm{m}$ and the cladding (refracting index, 1.41) with a thickness of $10 \mu \mathrm{m}$. A small part of the cladding was skinned off and the bared optical fiber, with a $5 \mathrm{~cm}$ long core section, was then dipped through the monolayer. LB films were transferred in the vertical mode at a constant surface pressure and a transfer rate of $20 \mathrm{~mm} \mathrm{~min}^{-1}$ onto the core of POFs. Polymerization experiments of the LB-deposited POFs were performed in a thermostated air bath by irradiation with a low-pressure $\mathrm{Hg}$ lamp at a distance of $7 \mathrm{~cm}$. Polymerization processes were traced by the use of the POF system as shows in Figure 1 and simultaneously by a multichannel photodetector (MCPD-1000, Otsuka Electronics Co., Ltd.). An LED source (MD927A, Anritsu Co., Ltd.) with a wavelength of $660 \mathrm{~nm}$ was connected with one end of the fiber. The light of LED passing through the fiber was picked up by an optical power meter (ML9002A, Anritsu Co., Ltd.). The refractive indices of cast films were measured at $20^{\circ} \mathrm{C}$ using an Abbe Refractometer (Shimadzu Co., Ltd.). The films were directly cast from $\mathrm{CCl}_{4}$ solutions on the prism of the refractometer.

\section{RESULTS AND DISCUSSION}

Figure 2 shows surface pressure $(\pi)$-area $(A)$ isotherms of $\mathbf{1}$ on pure water at $5^{\circ} \mathrm{C}$. The diacetylenic lipid $\mathbf{1}$ is found to provide a stable monolayer with a collapse pressure of $50 \mathrm{~m} \mathrm{~N} \mathrm{~m}^{-1}$ and a condensed solid phase at surface pressures $>25 \mathrm{~m} \mathrm{~N} \mathrm{~m}^{-1}$. To obtain information regarding molecular packing and crystallinity in the monolayer, the compression-expansion $\pi-A$ isotherms 

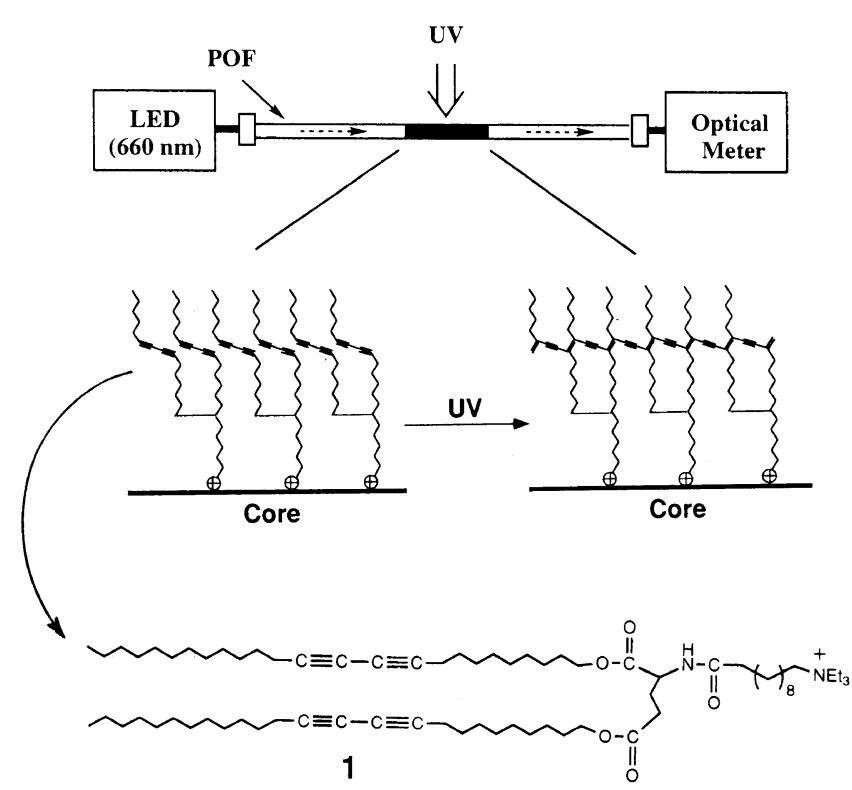

Figure 1. Schematic illustration for the POF system and the molecular structure of diacetylenic lipid $\mathbf{1}$.

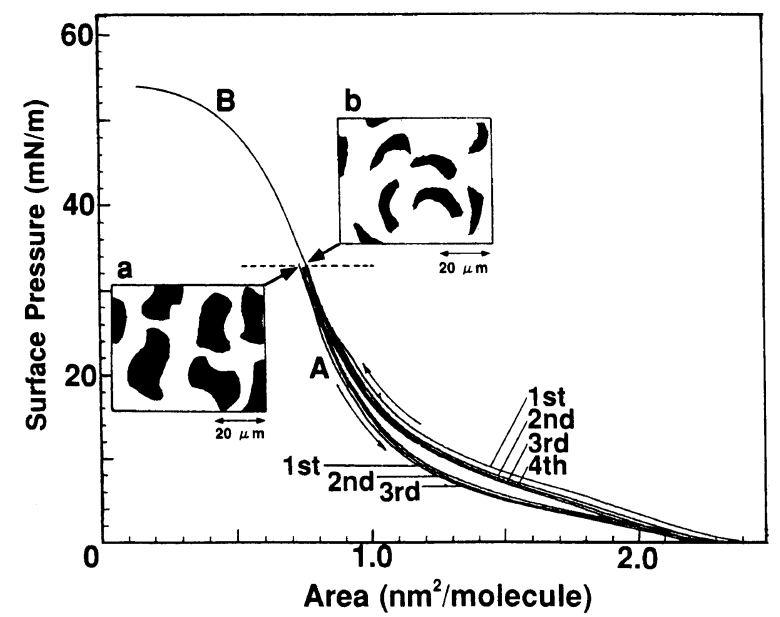

Figure 2. Surface pressure-area isotherms of 1 on pure water at $5^{\circ} \mathrm{C}$; compression-expansion cycle, (A); simple compression isotherm, (B). The inset shows fluorescence microscopic photographs taken at $32 \mathrm{mN}$ $\mathrm{m}^{-1}$ after repeated compression-expansion cycles (a) and after simple compression (b).

were measured repeatedly and shown in the same figure. A considerable difference is observed between the compression curve and that for expansion. In the expansion process, the $\pi-A$ curve provides a condensed phase at a lower surface pressure. It is also clearly seen from the figure that repeated compression-expansion cycle seems to bring about contraction of the monolayer with a slight shift of $\pi-A$ curves toward the smaller molecular area, suggesting that the packing density of molecules in the monolayer must be enhanced due to molecular interactions. Inset in Figure 2 shows photographs of fluorescence microscopic images of 1 monolayer taken at a constant surface pressure of $32 \mathrm{mN} \mathrm{m}^{-1}$ after four times compression-expansion cycles (A) and after simple compression (B). It should be noted that the bright areas in the photographs consist of molecules of $\mathbf{1}$ in liquid-expanded state homogeneously mixed with the fluorescent probe. For both monolayers, dark domains, in which molecules of $\mathbf{1}$ are in crystalline, are ob-

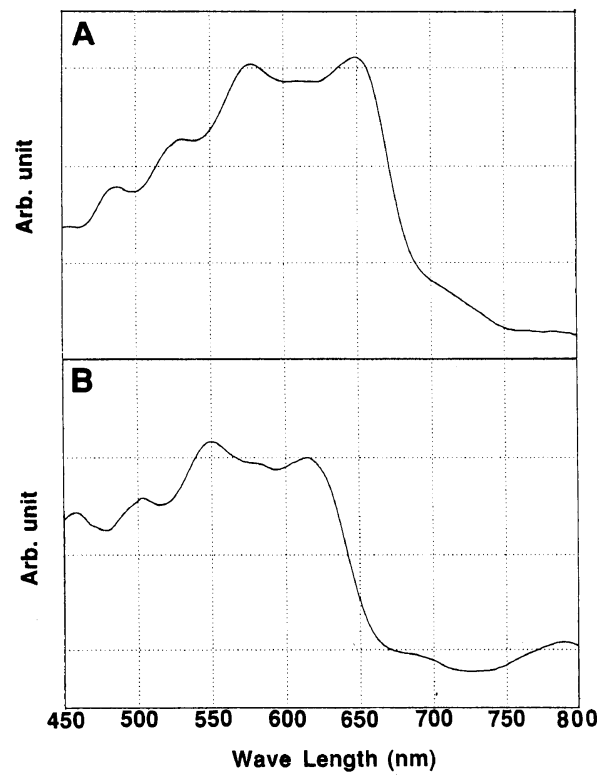

Figure 3. Reflection-absorption spectra of 1-LB films on the core of POF at $90 \mathrm{~s}-\mathrm{UV}$ irradiation. The $1-\mathrm{LB}$ films were transferred from monolayers at $32 \mathrm{~m} \mathrm{~N} \mathrm{~m}^{-1}$ after repeated compression-expansion cycles (A) and after simple compression (B).

served, and the domain size of the monolayer after repeated compression-expansion cycles (A) is larger than that after simple compression (B). This observation indicates that such a repeated compression-expansion of the monolayer would induce the formation of a well-grown crystalline domain.

Each monolayer (A or B) was subsequently transferred in the vertical mode at a surface pressure of $32 \mathrm{mN} \mathrm{m}^{-1}$ onto the core of POF. The transfer ratio $( \pm 15 \%)$ was close to unity both in the down-stroke and in the up-stroke mode. Figure 3 displays reflection-absorption spectra of the resulting LB films polymerized by $\mathrm{UV}$-irradiation for $90 \mathrm{~s}$ that was enough to complete polymerization. The absorption maxima of polydiacetylene are indicative of the effective conjugation length of the polymer chain and/or the order of the polymer structure. In studies of polydiacetylene LB films, two absorption maxima are usually observed. ${ }^{9}$ One is found between 600 and $640 \mathrm{~nm}$, which corresponds to the so-called blue polymer, and the other is between 500 and $550 \mathrm{~nm}$, which corresponds to the shorter or less conjugated red polymer. It should be noted that the (A)-LB film yields a more highly conjugated blue polymer with an absorption maximum of $650 \mathrm{~nm}$. On the other hand, the (B)-LB film gives mainly a red polymer with an absorption maximum of $540 \mathrm{~nm}$ although the existence of a less conjugated blue polymer with a wavelength of $620 \mathrm{~nm}$ is not excluded. These spectral features for the polymerized LB films prepared under different conditions are consistent with the monolayer properties such as monolayer crystallinity and $\pi-A$ isotherms.

Figure 4 shows a polymerization process of the (B)-LB film on POF followed by optical intensity (OI) of LED laser and absorbance at $660 \mathrm{~nm}$. The OI value is found to decrease steeply and to be leveled off at about $90 \mathrm{~s}$. The observed decrease in OI is supposed to stem from the refractive index variation of LB layers caused by formation of polydiacetylene due to polymerization, 


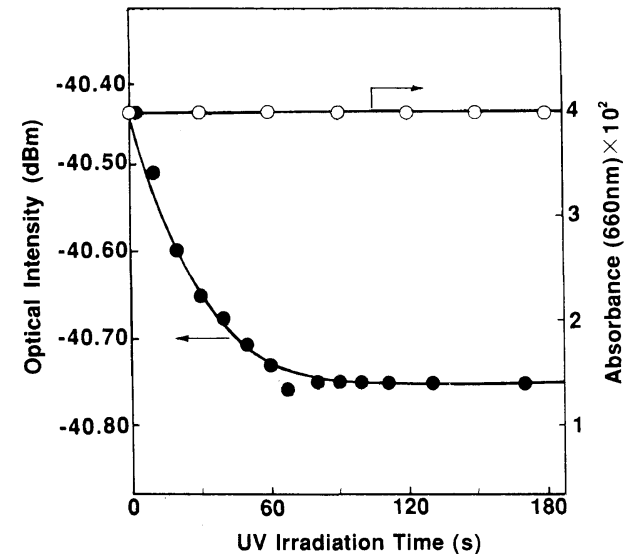

Figure 4. Changes in absorbance at $660 \mathrm{~nm}$ and OI of LED signal for the (B)-LB film upon UV irradiation.

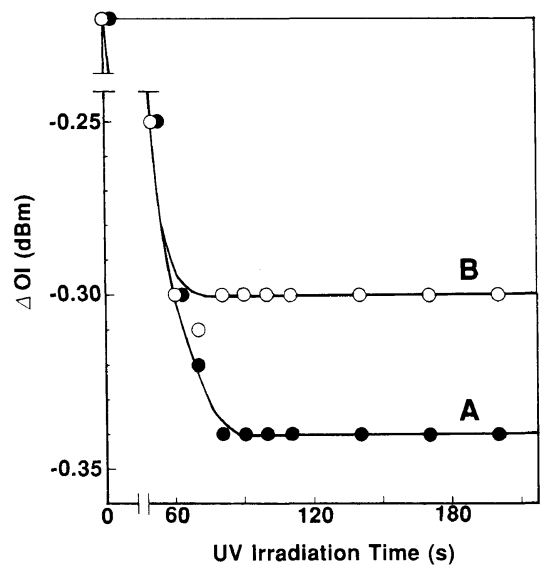

Figure 5. Comparison of $\Delta \mathrm{OI}$ changes with irradiation between the (A)- and (B)-LB films.

because there appears no absorption peak at around 660 $\mathrm{nm}$ during UV irradiation. In fact, the refractive indices for the cast film of 1 , prepared from its $\mathrm{CCl}_{4}$ solution, were measured at $20^{\circ} \mathrm{C}$ before and after polymerization ${ }^{10}$ to be 1.61 and 1.63 , respectively. The higher refractive index (1.63) observed after polymerization would induce lowering of the OI value since absorption of the light passing through the fiber into the LB layers deposited on the core might be suppressed, compared with that into the LB layers with a lower refractive index of 1.61 . Consequently, the OI value decreased with a progressive polymerization. Figure 5 shows a comparison of the relative change of $\mathrm{OI}(\Delta \mathrm{OI})$ caused by $\mathrm{UV}$ irradiation between (A)- and (B)-LB films. For both LB films, $\Delta \mathrm{OI}$ values are observed to decrease smoothly with irradiation and to be leveled off at around $90 \mathrm{~s}$. There is, however, a remarkable difference between the (A)- and (B)-LB films. The value of $\Delta \Delta$ OI for the (A)-LB film is considerably larger than that for the (B)-LB film, meaning that in the case of the (A)-LB film, which had an absorption peak at $650 \mathrm{~nm}$ very close to the wave length of LED, the OI change must arise from an absorption of LED light into the polydiacetylene LB layers as well as refractive index variation of the LB layers.

In conclusion, it has been found that polymerization processes of diacetylene LB film deposited on the core of POF can be sensitively monitored as an optical intensity change of LED light. Such a highly sensitive detection must be obtained due to a combined property of this POF system that can read out both changes in refractive indices and in color phases.

Acknowledgments. This work was supported by the project, "Nano Structure Hybrid Devices and Their Properties" at High Technology Center for Faculty of Engineering of Doshisha University. The project was supported by Grant-in-Aid from Doshisha University and The Ministry of Education, Science, Sports and Culture of Japan.

\section{REFERENCES AND NOTES}

1. M. Niwa, T. Yamamoto, and N. Higashi, Chem. Lett., 1603 (1990).

2. M. Niwa, T. Yamamoto, and N. Higashi, J. Chem. Soc., Chem. Commun., 444 (1991).

3. M. Niwa T. Yamamoto, and N. Higashi, J. Mater. Chem., 3, 703 (1993).

4. T. Kuo and D. F. O'Brien, J. Am. Chem. Soc., 110, 7571 (1988).

5. T. Kuo and D. F. O'Brien, Macromolecules, 23, 3225 (1990).

6. T. Kuo and D. F. O'Brien, Langmuir, 7, 584 (1991).

7. F. Saremi, E. Maassen, B. Tieke, G. Jordan, and W. Rammensee, Langmuir, 11, 1068 (1995).

8. T. Kim, R. M. Crooks, M. Tsen, and L. Sun, J. Am. Chem. Soc., 117, 3963 (1995)

9. (a) H.-J. Cantow, Ed., "Polydiacetylene," Springer Verlag, Berlin, 1984; (b) D. Bloor and R. R. Chance, "Polydiacetylenes. Advances in Polymer Science," Vol. 63, Martinus Nijhoff, Boston, MA, 1985.

10. The polymerization of the cast film of $\mathbf{1}$ was carried out by UV-irradiation at $20^{\circ} \mathrm{C}$ for $2 \mathrm{~h}$. The cast film after $2 \mathrm{~h}$-irradiation gave an absorption maximum at $620 \mathrm{~nm}$. 